Bull. Korean Math. Soc. 50 (2013), No. 5, pp. 1737-1751

http://dx.doi.org/10.4134/BKMS.2013.50.5.1737

\title{
A POINT COLLOCATION SCHEME FOR THE STATIONARY INCOMPRESSIBLE NAVIER-STOKES EQUATIONS
}

\author{
YONGSIK KIM
}

\begin{abstract}
An efficient and stable point collocation scheme based on a meshfree method is studied for the stationary incompressible NavierStokes equations. We describe the diffuse derivatives associated with the moving least square method. Using these diffuse derivatives, we propose a point collocation method to fit in solving the Navier-Stokes equations which improves the stability of the direct point collocation scheme. The convergence of the numerical solution is investigated from numerical examples. The driven cavity flow and the backward facing step flow are implemented for the reliability of the scheme. Also, the viscous flow on complicated geometry is successfully calculated such as the flow past a circular cylinder in duct.
\end{abstract}

\section{Introduction}

The objective of this paper is to propose a point collocation scheme for the stationary incompressible Navier-Stokes equations which is an extension of the previous work [11]. We proposed a direct point collocation schemes by a meshfree approximation which is called the fast moving least square reproducing kernel method (FMLSRK) [11].

Through a couple of decades, various meshfree approximations and algorithms have been studied $[3,4,5,6,8,13,14,15,16,17,18,19,21,22,25]$. While these methods are developed in the context of the Galerkin formulation, direct discretisation schemes such as point collocation methods were also proposed $[1,7,12,20,23]$. Most of meshfree methods share a main idea of making the shape functions which are related to the moving least square process. Those shape functions have certain reproducing property of polynomials, and that makes meshfree methods applicable to the collocation scheme. However, previous works on meshfree methods use the classical derivatives of basis to

Received December 19, 2012

2010 Mathematics Subject Classification. Primary 65N35, 76M25.

Key words and phrases. point collocation method, meshfree approximation, Navier-Stokes equations.

The work was supported by Basic Science Research Program through the National Research Foundation of Korea(NRF) funded by the Ministry of Education, Science and Technology(2011-0011855) and (313-2008-2-C00100). 
discretize partial differential equations, and that is a cause of excessive cost. In FMLSRK approximation, we use the diffuse derivatives of shape functions from the local interpolation, and the overall cost is remarkably reduced in the meshfree collocation schemes. The main difference between the FMLSRK and other meshfree approximation is the view on the derivatives of interpolation basis. In the process of obtaining interpolation basis, we interpret remnants as derivatives of interpolation basis.

In our previous work [11], we validated point collocation schemes for the Poisson equation and the Stokes equations. However, it is observed that there are certain instability phenomena for asymmetric Stokes problems. Further, the Navier-Stokes equations have intrinsic asymmetric property. Hence, we develop new collocation scheme which is suitable to the stationary incompressible Navier-Stokes equations. The main idea of the scheme is the constrained minimization procedure with the essential boundary condition as constraints. Introducing residual operators for momentum equation and divergence free condition, we define a square residual functional. Then, the numerical solution is the minimizer of the square residual functional with the essential boundary condition as constraints. In algebraic point of view, the proposed scheme is a kind of the least square process. Eventually, we solve a symmetric positive definite matrix even though original problem is asymmetric. While the description of the basic method follows the previous work [11], we need the concept of the dilation function [10] in calculating the flow on complicated geometry. We refer the work of Kim et al. [10] for further detail.

As numerical examples, convergence of relative $L^{2}, L^{\infty}$ errors were shown numerically for an exact solution of the Navier-Stokes equations. The driven cavity flow and the backward facing step flow are illustrated for the reliability of code. Furthermore, the flow in duct passing around a circular cylinder is also implemented to show the robustness of the scheme even on complicated geometry.

\section{Fast moving least square reproducing kernel approximation}

In this section, we briefly describe the FMLSRK approximation. The main difference between the FMLSRK and other meshfree methods is the way to obtain derivatives of shape functions. Derivatives of FMLSRK shape functions are obtained from the process of obtaining shape function itself without further cost. We interpret the limit of derivatives of local approximation as derivatives of global approximations. It can be explained as turning over the sequence of obtaining derivatives of shape functions. For the detail of FMLSRK, we refer [11]. Also we note that multi-index notation is used throughout the paper.

Let $\mathbf{P}_{m}(\mathbf{x})$ be the vector such that its elements are made by all of polynomials of order less than or equal to $m$ with $\mathbf{x} \in \mathbb{R}^{n}$. For example, if $n=2$ and $m=2$, then $\mathbf{P}_{m}(\mathbf{x})$ is $\left(1, x, y, x^{2}, x y, y^{2}\right)^{T}$. Suppose we have given node 
set $\Lambda=\left\{\mathbf{x}_{I} \in \Omega \mid I=1,2, \ldots, N P\right\}$ on the domain $\Omega \subset \mathbb{R}^{n}$. With a compactly supported continuous non-negative window function $\Phi$, the resulting shape functions of FMLSRK are defined as the following:

$$
\Psi_{I}^{[\alpha]}(\mathbf{x}) \equiv \frac{\alpha !}{\rho^{|\alpha|}} \mathbf{e}_{\alpha}^{T} M^{-1}(\mathbf{x}) \mathbf{P}_{m}\left(\frac{\mathbf{x}_{I}-\mathbf{x}}{\rho}\right) \Phi_{\rho}\left(\mathbf{x}_{I}-\mathbf{x}\right) .
$$

Here, $\Phi_{\rho}(\mathbf{x}-\overline{\mathbf{x}})=\frac{1}{\rho^{n}} \Phi\left(\frac{\mathbf{x}-\overline{\mathbf{x}}}{\rho}\right)$ and $M$ is the conventional moment matrix as the following;

$$
M(\mathbf{x}) \equiv \sum_{I=1}^{N P} \mathbf{P}_{m}\left(\frac{\mathbf{x}_{I}-\overline{\mathbf{x}}}{\rho_{\overline{\mathbf{x}}}}\right) \mathbf{P}_{m}^{T}\left(\frac{\mathbf{x}_{I}-\overline{\mathbf{x}}}{\rho_{\overline{\mathbf{x}}}}\right) \Phi_{\rho}\left(\mathbf{x}_{I}-\mathbf{x}\right) .
$$

Also $\alpha=\left(\alpha_{1}, \ldots, \alpha_{n}\right)$ is multi index and $\mathbf{e}_{\alpha}$ is the $\alpha$-th unit vector in $\mathbb{R}^{\frac{(n+m) !}{n ! m !}}$. We note that only continuity is required for the window function instead of differentiability. For example, if $n=2$ and $m=2$, explicit shape functions are the following.

$$
\left(\begin{array}{l}
\Psi_{I}^{[(0,0)]}(\mathbf{x}) \\
\Psi_{I}^{[(1,0)]}(\mathbf{x}) \\
\Psi_{I}^{[(0,1)]}(\mathbf{x}) \\
\Psi_{I}^{[(2,0)]}(\mathbf{x}) \\
\Psi_{I}^{[(1,1)]}(\mathbf{x}) \\
\Psi_{I}^{[(0,2)]}(\mathbf{x})
\end{array}\right)=\left(\begin{array}{cccccc}
1 & 0 & 0 & 0 & 0 & 0 \\
0 & \frac{1}{\rho} & 0 & 0 & 0 & 0 \\
0 & 0 & \frac{1}{\rho} & 0 & 0 & 0 \\
0 & 0 & 0 & \frac{2}{\rho^{2}} & 0 & 0 \\
0 & 0 & 0 & 0 & \frac{1}{\rho^{2}} & 0 \\
0 & 0 & 0 & 0 & 0 & \frac{2}{\rho^{2}}
\end{array}\right) M^{-1}(\mathbf{x})\left(\begin{array}{c}
1 \\
\frac{x_{I}-x}{\rho} \\
\frac{y-y}{\rho} \\
\frac{\left(x_{I}-x\right)^{2}}{\rho^{2}} \\
\frac{\left(x_{I}-x\right)\left(y_{I}-y\right)}{\rho^{2}} \\
\frac{\left(y_{I}-y\right)^{2}}{\rho^{2}}
\end{array}\right) \Phi_{\rho}\left(\mathbf{x}_{I}-\mathbf{x}\right)
$$

If $\alpha$ is zero, $\Psi_{I}^{[\alpha]}$ s are usual shape functions in meshfree approximation. Otherwise, i.e., $\alpha \neq \mathbf{0}, \Psi_{I}^{[\alpha]}$ corresponds to $\frac{\partial \Psi_{I}^{[0]}}{\partial \mathbf{x}^{\alpha}}$. Hence, there is no more step to produce derivatives of shape functions.

Defining the global interpolation operator as $D_{m}^{\rho, \alpha} u(\mathbf{x})=\sum_{I=1}^{N P} \Psi_{I}^{[\alpha]}(\mathbf{x}) u\left(\mathbf{x}_{I}\right)$, the general consistency theorem and the interpolation theorem by FMLSRK are obtained (see [11]).

Theorem 2.1. Suppose that $u(\mathbf{x})$ is a polynomial of the order less than or equal to $m$. Then the interpolation operator $D_{m}^{\rho, \alpha}$ produces $D^{\alpha} u(\mathbf{x})$, i.e.,

$$
D_{m}^{\rho, \alpha} u(\mathbf{x})=D^{\alpha} u(\mathbf{x})
$$

for any $\alpha,|\alpha| \leq m$.

Theorem 2.2. Assume the window function $\Phi(\mathbf{x}) \in C_{0}^{0}\left(\mathbb{R}^{n}\right)$ and $v(\mathbf{x}) \in$ $C^{m+1}(\bar{\Omega})$, where $\Omega$ is a bounded open set in $\mathbb{R}^{n}$. Let $\Lambda=\left\{\mathbf{x}_{I} \mid I=1, \ldots, N P\right\}$ be a regular node set. Suppose the boundary of $\Omega$ is smooth and supp $\Psi_{I}^{[\mathbf{0}]} \cap \bar{\Omega}$ is convex for each $I$.

If $m$ and $p$ satisfy $m>\frac{n}{p}-1$, then the following interpolation estimate holds (2) $\left\|D^{\beta} v-D_{m}^{\rho, \beta} v\right\|_{L^{p}(\Omega)} \leq C(m) \rho^{m+1-|\beta|}\|v\|_{W^{m+1, p}(\Omega)}$ for all $0 \leq|\beta| \leq m$. 
For convenience of further study, a linear operator will be defined between vector spaces which are generated by shape functions.

Definition 1. We define the finite dimensional vector space $H_{\rho}^{\alpha}$. The $\alpha$-th shape functions $\Psi_{I}^{[\alpha]}(\mathbf{x})$ 's make the vector space

$$
H_{\rho}^{\alpha}=\left\{\sum_{\mathbf{x}_{I} \in \Lambda} u_{I} \Psi_{I}^{[\alpha]}(\mathbf{x}) \mid u_{I} \in \mathbb{R}\right\}, \quad|\alpha| \leq m,
$$

and we define the linear operator $D_{m, h}^{\rho, \alpha}: H_{\rho}^{\mathbf{0}} \rightarrow H_{\rho}^{\alpha}$ such that

$$
D_{m, h}^{\rho, \alpha}\left(\Psi_{I}^{[\mathbf{0}]}(\mathbf{x})\right)=\Psi_{I}^{[\alpha]}(\mathbf{x}) \text { for all } I=1,2, \ldots, N P .
$$

We call it the $\alpha$-th discrete differential operator.

By definition, we see that $D_{m, h}^{\rho, 0}$ is the identity operator. On the other hand, the role of $\Psi_{I}^{[\alpha]}(\mathbf{x})$ is to take the place of the direct derivative $D^{\alpha} \Psi_{I}^{[\mathbf{0}]}(\mathbf{x})$ of the shape function $\Psi_{I}^{[\mathbf{0}]}(\mathbf{x})$. Also, we note that there is a following relation,

$$
D_{m}^{\rho, \alpha}=D_{m, h}^{\rho, \alpha} \circ D_{m}^{\rho, \mathbf{0}}: C^{0}(\bar{\Omega}) \rightarrow H_{\rho}^{\alpha},
$$

where $C^{0}(\bar{\Omega})$ is the set of all continuous functions up to boundary of $\Omega$.

Remark 2.3. It is natural to extend the above method using the dilation function. The constant dilation parameter $\rho$ can be replaced with the continuous dilation function $\rho_{\mathbf{x}}$. However, it is difficult to adopt a dilation function to meshfree methods using exact evaluation of derivatives. In [10], an algorithm to make continuous dilation function from a given node distribution was proposed. The interpolation theorem (Theorem 2) is also valid for the case of using the dilation function. In that case, $\rho$ is replaced with $\max _{\mathbf{x} \in \Omega} \rho_{\mathbf{x}}$.

\section{Preconditioned point collocation scheme using dilation function}

The pre-conditioned point collocation scheme is quite natural in FMLSRK. For simplicity, we choose the Poisson equation as an example to explain the pre-conditioned point collocation scheme. Using FMLSRK approximation, we interpolate the solution $u(\mathbf{x})$ of a given partial differential equation such as

$$
u \approx U=\sum_{J=1}^{N P} u_{J} \Psi_{J}^{[\mathbf{0}]}\left(\mathbf{x}, \rho_{\mathbf{x}}\right) \in H_{\rho_{\mathbf{x}}}^{\mathbf{0}},
$$

where $u_{I}$ will be determined by the governing equation. Then Theorem 2.1 and Definition 1 lead us to discretize the derivatives of $u(\mathbf{x})$ as follows:

$$
D^{\beta} u \approx D_{m, h}^{\rho_{\mathbf{x}}, \beta} U=\sum_{J=1}^{N P} u_{J} \Psi_{J}^{[\beta]}\left(\mathbf{x}, \rho_{\mathbf{x}}\right) \in H_{\rho_{\mathbf{x}}}^{\beta} \quad \text { for } \quad 0<|\beta| \leq m .
$$

We are going to solve strong form of the governing equation like finite difference method (FDM). However, we find some difference from this method. First, no 
grid structure is needed. Second, it has interpolation not only for the solution but also for derivatives of solution up to the order of the consistency. Third, it is easy to make higher order scheme by increasing the order of the basis polynomial vector $\mathbf{P}_{m}$.

Now, let $\Omega$ be a bounded domain in $\mathbb{R}^{n}$ with its boundary $\Gamma$. Here we only consider $n=2$, since the scheme has no dependency of dimension. We consider the Poisson equation:

$$
\begin{aligned}
-\Delta u & =f, & & \text { in } \Omega \\
u & =g, & & \text { on } \Gamma_{D} \\
\frac{\partial u}{\partial n} & =h, & & \text { on } \Gamma_{N}
\end{aligned}
$$

where $\partial \Omega=\Gamma_{D} \cup \Gamma_{N}, \Gamma_{D} \cap \Gamma_{N}=\phi$.

We propose a pre-conditioned point collocation scheme in terms of the approximations (5) and (6) such as

$$
U(\mathbf{x})=\sum_{\mathbf{x}_{J} \in \Lambda} u_{J} \Psi_{J}^{[(0,0)]}\left(\mathbf{x}, \rho_{\mathbf{x}}\right) \in H_{\rho_{\mathbf{x}}}^{\mathbf{0}}
$$

$$
-\sum_{\mathbf{x}_{J} \in \Lambda} u_{J}\left(\rho_{\mathbf{x}_{I}}^{2} \Delta^{h} \Psi_{J}\left(\mathbf{x}_{I}, \rho_{\mathbf{x}_{I}}\right)\right)=\rho_{\mathbf{x}_{I}}^{2} f\left(\mathbf{x}_{I}\right) \quad \text { for all } \quad \mathbf{x}_{I} \in \Lambda_{i},
$$

$$
\sum_{\mathbf{x}_{J} \in \Lambda} u_{I} \Psi_{J}\left(\mathbf{x}_{I}, \rho_{\mathbf{x}_{I}}\right)=g\left(\mathbf{x}_{I}\right) \text { for all } \quad \mathbf{x}_{I} \in \Lambda_{d}
$$

(12) $\sum_{\mathbf{x}_{J} \in \Lambda} u_{J}\left(\rho_{\mathbf{x}_{I}} \nabla^{h} \Psi_{J}\left(\mathbf{x}_{I}, \rho_{\mathbf{x}_{I}}\right)\right) \cdot \mathbf{n}\left(\mathbf{x}_{I}\right)=\rho_{\mathbf{x}_{I}} h\left(\mathbf{x}_{I}\right) \quad$ for all $\quad \mathbf{x}_{I} \in \Lambda_{n}$,

$$
\begin{aligned}
& \Delta^{h} \Psi_{J}\left(\mathbf{x}, \rho_{\mathbf{x}}\right) \equiv \Psi_{J}^{[(2,0)]}\left(\mathbf{x}, \rho_{\mathbf{x}}\right)+\Psi_{J}^{[(0,2)]}\left(\mathbf{x}, \rho_{\mathbf{x}}\right), \\
& \left.\nabla^{h} \Psi_{J}\left(\mathbf{x}, \rho_{\mathbf{x}}\right) \equiv \Psi_{J}^{[(1,0)]}\left(\mathbf{x}, \rho_{\mathbf{x}}\right), \Psi_{J}^{[(0,1)]}\left(\mathbf{x}, \rho_{\mathbf{x}}\right)\right),
\end{aligned}
$$

where $\Lambda=\Lambda_{i} \cup \Lambda_{d} \cup \Lambda_{n}$, and $\Lambda_{i}, \Lambda_{d}$ and $\Lambda_{n}$ are sets of interior nodes, Dirichlet boundary nodes and Neumann boundary nodes, respectively. Here $\mathbf{n}\left(\mathbf{x}_{I}\right)$ is the outward unit normal vector at $\mathbf{x}_{I} \in \Lambda_{n}$. In this case, at least the second order approximated derivatives are essential. Thus, we choose the order $m$ of basis polynomial $\mathbf{P}_{m}$ greater than or equal to 2 .

\section{A point collocation scheme for the stationary incompressible Navier-Stokes equations}

In this section, we propose a collocation scheme by FMLSRK for the stationary incompressible Navier-Stokes equations in $\mathbb{R}^{n}(n=2,3)$,

$$
\begin{aligned}
-\nu \Delta \mathbf{u}+(\mathbf{u} \cdot \nabla) \mathbf{u}+\nabla p & =\mathbf{f}, & & \text { in } \Omega \\
\nabla \cdot \mathbf{u} & =0, & & \text { in } \Omega \\
\mathbf{u} & =\mathbf{g}, & & \text { on } \partial \Omega
\end{aligned}
$$


where the boundary value $\mathrm{g}$ satisfies the compatibility condition for solvability:

$$
\int_{\partial \Omega} \mathbf{g} \cdot \mathbf{n} d \Gamma=0
$$

Here the pressure is determined up to constant.

What we propose for the Navier-Stokes flow is adopting two nested node sets for velocity and pressure. The fine node set is for the velocity, while the course node set is for the pressure. In $[4,5]$, authors adopted same kind of node sets for the incompressible Navier-Stokes equations with Galerkin formulation. Also in [11], same kind of node sets are used for the stationary incompressible Stokes equations with point collocation scheme. For the unique solvability of the viscous incompressible flows, a pair of node sets satisfying LBB-like condition (i.e., similar with LBB condition) seems to be inevitable.

Since there is no essential difference of this point collocation scheme to the space dimension $n$, we concentrate only for 2 -dimensional problems. Let $\Omega$ be the 2-dimensional region of fluid, and $\mathbf{x}_{I}^{V}$ 's and $\mathbf{x}_{K}^{P}$ 's be chosen as node points in $\bar{\Omega}$ for velocity and pressure, respectively. Also, suppose $\Lambda^{V}$ and $\Lambda^{P}$ are sets of $\mathbf{x}_{I}^{V}$ 's and $\mathbf{x}_{K}^{P}$ 's, respectively. Let us denote $\left\{\Psi_{J}\right\}_{\mathbf{x}_{J}^{V} \in \Lambda^{V}}$ and $\left\{\bar{\Psi}_{K}^{P}\right\}_{\mathbf{x}_{K}^{P} \in \Lambda^{P}}$ are sets of shape functions corresponding to velocity nodes and pressure nodes, respectively. Let $\mathbf{U}=(U, V)$ and $P$ be the numerical solution by FMLSRK, for the stationary incompressible Navier-Stokes equations (13), (14) and (15) as follows:

$$
\begin{aligned}
& \mathbf{U}(\mathbf{x})=\sum_{\mathbf{x}_{J}^{V} \in \Lambda^{V}} \mathbf{U}_{J} \Psi_{J}^{[(0,0)]}(\mathbf{x}), \\
& P(\mathbf{x})=\sum_{\mathbf{x}_{K}^{P} \in \Lambda^{P}} P_{K} \bar{\Psi}_{K}^{[(0,0)]}(\mathbf{x}),
\end{aligned}
$$

where $\mathbf{U}_{J}=\left(U_{J}, V_{J}\right)$.

The discrete differential operators are used for the discretization of the partial differential equations, which are defined in Definition 1. In short, the following approximations of derivatives are used,

$$
\begin{aligned}
& D^{\alpha} \mathbf{U}(\mathbf{x}) \approx D_{m, h}^{\rho, \alpha} \mathbf{U}(\mathbf{x})=\sum_{\mathbf{x}_{J}^{V} \in \Lambda^{V}} \mathbf{U}_{J} \Psi_{J}^{[\alpha]}(\mathbf{x}), \\
& D^{\alpha} P(\mathbf{x}) \approx D_{m, h}^{\rho, \alpha} P(\mathbf{x})=\sum_{\mathbf{x}_{K}^{P} \in \Lambda^{P}} P_{K} \bar{\Psi}_{K}^{[\alpha]}(\mathbf{x}),
\end{aligned}
$$

where $\mathbf{U}_{J}=\left(U_{J}, V_{J}\right)$ and $|\alpha| \leq m$.

For simplicity, let us define the discrete differential symbols $\nabla^{h}$ and $\Delta^{h}$ induced from Definition 1 as follows:

$$
\begin{aligned}
& \Delta^{h} \Psi_{J}=\Psi_{J}^{[(2,0)]}+\Psi_{J}^{[(0,2)]}, \\
& \nabla^{h} \Psi_{J}=\left(\Psi_{J}^{[(1,0)]}, \Psi_{J}^{[(0,1)]}\right), \\
& \nabla \cdot \bar{\Psi}_{J}=\bar{\Psi}_{J}^{[(1,0)]}+\bar{\Psi}_{J}^{[(0,1)]} .
\end{aligned}
$$


Now, define the error residual operators on $\Omega$ for the Navier-Stokes equations, one for the momentum equations and another for the incompressibility:

$$
\begin{aligned}
\mathcal{R}_{\mathcal{M}}(\mathbf{w}, \mathbf{u}, p) & \equiv-\nu A^{h}(\mathbf{u})+B^{h}(\mathbf{w}, \mathbf{u})+C^{h}(p)-\mathbf{f}, \\
\mathcal{R}_{\mathcal{C}}(\mathbf{u}) & \equiv C_{*}^{h}(\mathbf{u}),
\end{aligned}
$$

where $A^{h}(\cdot), B^{h}(\cdot, \cdot, \cdot), C^{h}(\cdot)$ and $C_{*}^{h}(\cdot)$ are the approximate differential operators defined as the following:

$$
\begin{aligned}
A^{h}(\mathbf{u}) & =\sum_{\mathbf{x}_{J}^{V} \in \Lambda^{V}} \mathbf{U}_{J} \Delta^{h} \Psi_{J}, \\
B^{h}(\mathbf{w}, \mathbf{u}) & =\sum_{\mathbf{x}_{J}^{V} \in \Lambda^{V}} \mathbf{U}_{J}\left(\mathbf{W} \cdot \nabla^{h} \Psi_{J}\right), \\
C^{h}(p) & =\sum_{\mathbf{x}_{K}^{P} \in \Lambda^{P}} P_{K} \nabla^{h} \bar{\Psi}_{K}, \\
C_{*}^{h}(\mathbf{u}) & =\sum_{\mathbf{x}_{J}^{V} \in \Lambda^{V}} \mathbf{U}_{J} \cdot \nabla^{h} \Psi_{J},
\end{aligned}
$$

where $\mathbf{W}$ is the $(0,0)$-th approximation of $\mathbf{w}$ with respect to velocity shape functions $\Psi_{J}$ 's.

Let us define the square residual functional associated with node sets $\Lambda^{V}$ and $\Lambda^{P}$ :

$$
\mathcal{R}(\mathbf{W}, \mathbf{U}, P)=\sum_{\mathbf{x}_{J}^{V} \in \Lambda^{V}} \frac{1}{2} \mathcal{R}_{\mathcal{M}}(\mathbf{W}, \mathbf{U}, P)^{2}\left(\mathbf{x}_{J}^{V}\right)+\sum_{\mathbf{x}_{K}^{P} \in \Lambda^{P}} \frac{1}{2} \mathcal{R}_{\mathcal{C}}(\mathbf{U})^{2}\left(\mathbf{x}_{K}^{P}\right) .
$$

Now we consider the constrained minimization problem of $\mathcal{R}(\mathbf{U}, \mathbf{U}, P)$ with respect to $(\mathbf{U}, P)$ :

- $\operatorname{Minimum}_{(\mathbf{U}, P)} \mathcal{R}(\mathbf{U}, \mathbf{U}, P)$

subjected to $\left.\mathbf{U}\right|_{\Gamma}=\mathbf{g}$ and $P\left(\mathbf{x}_{J_{0}}\right)=p_{0}$ where $\Gamma$ is the boundary of $\Omega$, the point $\mathbf{x}_{J_{0}} \in \Lambda^{P}$ is a chosen point among pressure nodes and $p_{0}$ is some fixed value for pressure.

The last constraint plays a role in determining the pressure uniquely. It is known that the pressure is determined up to constant in mathematical theory of fluid.

To obtain the minimizer of the problem, the successive iteration scheme is used for the Navier-Stokes equations, since it is non-linear functional.

- STEP 0: Choose $\mathbf{U}^{(n)}$ satisfying boundary condition.

STEP 1: Find the minimizer $\left(\mathbf{U}^{(n+1)}, P^{(n+1)}\right)$, i.e., $\mathcal{R}\left(\mathbf{U}^{(n)}, \mathbf{U}^{(n+1)}, P^{(n+1)}\right)=\operatorname{Minimize}_{(\mathbf{U}, P)} \mathcal{R}\left(\mathbf{U}^{(n)}, \mathbf{U}, P\right)$ subjected to $\left.\mathbf{U}^{(n+1)}\right|_{\Gamma}=\mathbf{g}$ and $P\left(\mathbf{x}_{J_{0}}\right)=p_{0}$.

- STEP 2: If $\left\|\mathbf{U}^{(n+1)}-\mathbf{U}^{(n)}\right\|_{L^{2}}<\epsilon$, then STOP. Otherwise, set $n=n+1$ and go to STEP 0. Here, $\epsilon>0$ is a tolerance for successive error. 


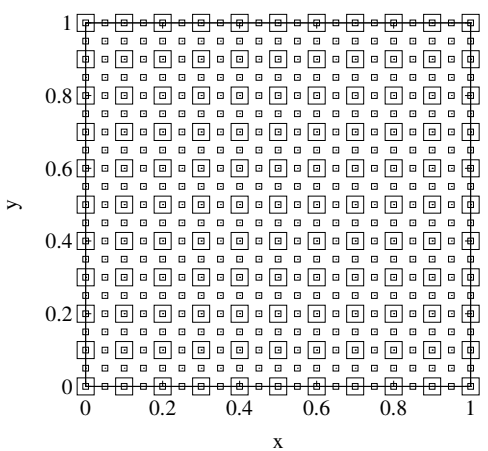

FiguRE 1. Node distributions of velocity and pressure for the Navier-Stokes flow: velocity node $(\square)$ and pressure node $(\square)$

\section{Numerical examples}

Four numerical examples are presented in this section. Except the flow in the backward-facing step, it is assumed that $\nu=\frac{1}{100}$, which is equivalent to $R e=100$, through examples. Through all numerical calculations, we choose the window function of the form

$$
\Phi(\mathbf{x})= \begin{cases}(1-\|\mathbf{x}\|)^{4}, & \text { when }\|\mathbf{x}\|<1, \\ 0, & \text { otherwise. }\end{cases}
$$

The first example is on the convergence rate of exact solution for the NavierStokes equations. The domain of the fluid is 2-dimensional unit square $\Omega=$ $[0,1] \times[0,1]$. The exact solution $\mathbf{u}=(u, v)$ and $p$ of the Navier-Stokes equations (13), (14), (15) and (16) are chosen such that

$$
\begin{aligned}
& u=\sin ^{3} \pi x \sin ^{2} \pi y \cos \pi y, \\
& v=-\sin ^{2} \pi x \sin ^{3} \pi y \cos \pi x, \\
& p=x^{2}-y^{2} .
\end{aligned}
$$

Then the external force $\mathbf{f}$ is calculated through the Navier-Stokes equations (13).

Two node sets $\Lambda^{V}$ and $\Lambda^{P}$ are used for the velocity and the pressure respectively, for solvability of the discrete equations. In Figure 1, the velocity nodes are located at points where the gradient of pressure shape functions are steep. It makes the condition number of the stiffness matrix smaller. To set the Dirichlet boundary condition as constraint, we employ so called d'Alembert's principle (see [9]). Let the solution of the $(n+1)$-th step of the algorithm in the previous section be $\left(\mathbf{U}^{(n+1)}, P^{(n+1)}\right)$. Then we have the representation formula

$$
\mathbf{U}^{(n+1)}(\mathbf{x})=\sum_{\mathbf{x}_{J}^{V} \in \Lambda^{V}} \mathbf{U}_{J}^{(n+1)} \Psi_{J}^{[(0,0)]}(\mathbf{x}),
$$




$$
P^{(n+1)}(\mathbf{x})=\sum_{\mathbf{x}_{K}^{P} \in \Lambda^{P}} P_{K}^{(n+1)} \bar{\Psi}_{K}^{[(0,0)]}(\mathbf{x}) .
$$

For simplicity, assume that the set of boundary nodes for velocity is $\Gamma^{d}=$ $\left\{\mathbf{x}_{J}^{V} \in \Lambda^{V} \mid 1 \leq J \leq N_{b d y}\right\}$, where $N_{b d y}$ is the number of boundary nodes. Since $\mathbf{U}^{(n+1)}\left(\mathbf{x}_{J}^{V}\right)=\sum_{\mathbf{x}_{I}^{V} \in \Lambda^{V}} \mathbf{U}_{I}^{(n+1)} \Psi_{I}^{[(0,0)]}\left(\mathbf{x}_{J}^{V}\right)=\mathbf{g}\left(\mathbf{x}_{J}^{V}\right)$ for $\mathbf{x}_{J}^{V} \in \Gamma^{d}$, we have the following relation between nodal variables and essential boundary conditions on the boundary of the domain,

$$
\left(\begin{array}{c}
\mathbf{U}_{b d y}^{(n+1)} \\
\mathbf{U}_{i n t}^{(n+1)}
\end{array}\right)=\mathbf{A}^{-1}\left(\begin{array}{c}
\mathbf{G} \\
\mathbf{U}_{\text {int }}^{(n+1)}
\end{array}\right)
$$

Here matrices written above are defined as followings, while $N^{V}$ is the number of velocity nodes:

$$
\mathbf{U}_{b d y}^{(n+1)}=\left(\begin{array}{c}
\mathbf{U}_{1}^{(n+1)} \\
\vdots \\
\mathbf{U}_{N_{b d y}}^{(n+1)}
\end{array}\right), \quad \mathbf{U}_{i n t}^{(n+1)}=\left(\begin{array}{c}
\mathbf{U}_{N_{b d y}+1}^{(n+1)} \\
\vdots \\
\mathbf{U}_{N^{V}}^{(n+1)}
\end{array}\right), \quad \text { and } \quad \mathbf{G}=\left(\begin{array}{c}
\mathbf{g}\left(\mathbf{x}_{1}^{V}\right) \\
\vdots \\
\mathbf{g}\left(\mathbf{x}_{N_{b d y}}^{V}\right)
\end{array}\right)
$$

The matrix $\mathbf{A}$ is the following form

$$
\mathbf{A}=\left(\begin{array}{cc}
\Psi_{B B} & \Psi_{B I} \\
\mathbf{0} & \mathbf{I}
\end{array}\right)
$$

with defining $\Psi_{B B}$ and $\Psi_{B I}$ as followings,

and

$$
\Psi_{B B}=\left(\begin{array}{ccc}
\Psi_{1}^{[(0,0)]}\left(\mathbf{x}_{1}^{V}\right) & \cdots & \Psi_{N_{b d y}}^{[(0,0)]}\left(\mathbf{x}_{1}^{V}\right) \\
\vdots & \ddots & \vdots \\
\Psi_{1}^{[(0,0)]}\left(\mathbf{x}_{N_{b d y}}^{V}\right) & \cdots & \Psi_{N_{b d y}}^{[(0,0)]}\left(\mathbf{x}_{N_{b d y}}^{V}\right)
\end{array}\right)
$$

$$
\Psi_{B I}=\left(\begin{array}{ccc}
\Psi_{N_{b d y}+1}^{[(0,0)]}\left(\mathbf{x}_{1}^{V}\right) & \cdots & \Psi_{N^{V}}^{[(0,0)]}\left(\mathbf{x}_{1}^{V}\right) \\
\vdots & \ddots & \vdots \\
\Psi_{N_{b d y}+1}^{[(0,0)]}\left(\mathbf{x}_{N_{b d y}}^{V}\right) & \cdots & \Psi_{N^{V}}^{[(0,0)]}\left(\mathbf{x}_{N_{b d y}}^{V}\right)
\end{array}\right)
$$

The initial solution of the scheme is chosen as a solution of the Stokes equations with same force and same boundary condition. The minimizer of the residual functional $\mathcal{R}\left(\mathbf{U}^{(n)}, \mathbf{U}^{(n+1)}, P^{(n+1)}\right)$ have 0 Fréchet derivative value. Hence we have

$$
\mathbf{M}\left(\mathbf{U}^{(n)}\right)\left(\begin{array}{ccc}
\Psi_{B B}{ }^{-1} & -\Psi_{B B}{ }^{-1} \Psi_{B I} & \mathbf{0} \\
\mathbf{0} & \mathbf{I} & \mathbf{0} \\
\mathbf{0} & \mathbf{0} & \mathbf{I}
\end{array}\right)\left(\begin{array}{c}
\mathbf{G} \\
\mathbf{U}_{i n t}^{(n+1)} \\
\mathbf{P}^{(n+1)}
\end{array}\right)=\left(\begin{array}{c}
\mathbf{F}_{b d y} \\
\mathbf{F}_{i n t} \\
\mathbf{0}
\end{array}\right)
$$

where

$$
\mathbf{M}\left(\mathbf{U}^{(n)}\right)=\left(\begin{array}{cc}
-\nu \Delta^{h} \Psi_{J}\left(\mathbf{x}_{I}^{V}\right)+\mathbf{U}^{(n)} \cdot \nabla^{h} \Psi_{J}\left(\mathbf{x}_{I}^{V}\right) & \nabla^{h} \Psi_{J}\left(\mathbf{x}_{I}^{V}\right) \\
\nabla \cdot \bar{\Psi}_{J}\left(\mathbf{x}_{K}^{P}\right) & \mathbf{0}
\end{array}\right)
$$




$$
\mathbf{P}^{(n+1)}=\left(\begin{array}{c}
P_{1}^{(n+1)} \\
\vdots \\
P_{N^{P}}^{(n+1)}
\end{array}\right)
$$

Eventually, after passing over constraint to force terms, over-determined linear system is obtained. By multiplying the transpose of the stiffness matrix to both sides, the minimizer is obtained.

The convergence rates of relative $L^{2}$ errors and $L^{\infty}$ errors are illustrated in Figure 2. Four different uniform node sets are used. Numbers of velocity nodes and pressure nodes are $(121,36),(441,121),(1681,441),(6561,1681)$ respectively. We tested the problem with two types of shape functions: the second order basis $(m=2)$ and the fourth order basis $(m=4)$. The figure shows that the convergence rate for the pressure has the same order as that for the velocity. In general, the order of $L^{2}$ convergence rate for pressure is equal to that of $H^{1}$ convergence rate for velocity in FEM. In this point of view, it seems that this result shows some benefits of this scheme.

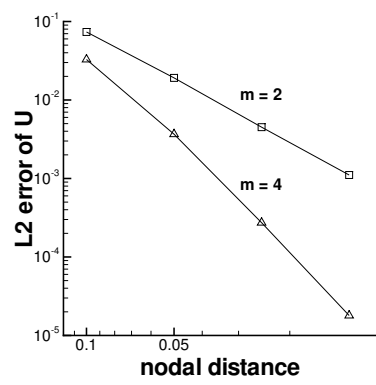

(a) $\frac{\|\mathbf{U}-\mathbf{u}\|_{L^{2}}}{\|\mathbf{u}\|_{L^{2}}}$

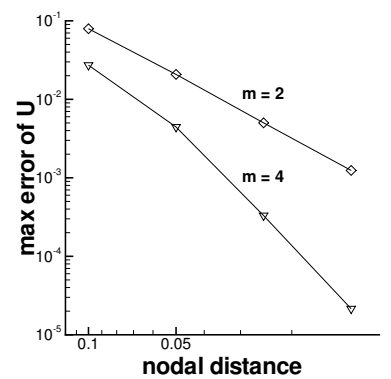

(c) $\frac{\|\mathbf{U}-\mathbf{u}\|_{L} \infty}{\|\mathbf{u}\|_{L} \infty}$

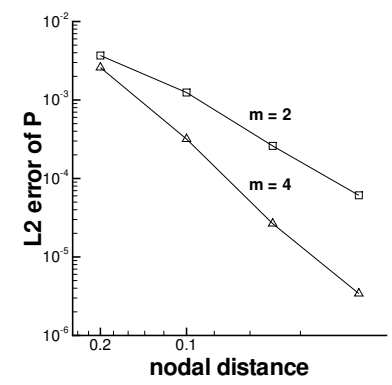

(b) $\frac{\|P-p\|_{L^{2}}}{\|p\|_{L^{2}}}$

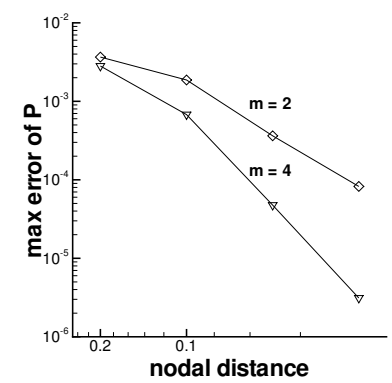

(d) $\frac{\|P-p\|_{L^{2} \infty}}{\|p\|_{L^{\infty}}}$

FiguRE 2. Relative errors $(R e=100)$ 
The second example is on the lid-driven cavity flow. The lid-driven cavity problem has long been used as a test for new methods. We used same type of node sets as in the first example. For the velocity, $51 \times 51$ nodes are used, while $26 \times 26$ nodes are used for the pressure. Unit $x$-velocity is assigned on the upper wall while 0 velocity is assigned on others. The plot of the streamlines, the contour of the vorticity and the contour lines of the pressure are plotted in Figure 3 .

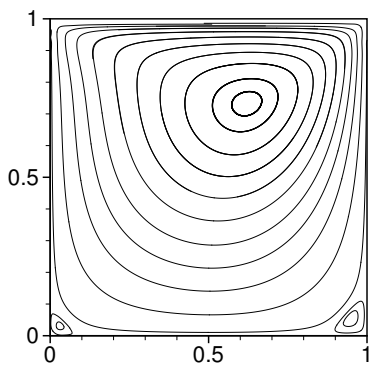

(a) streamlines

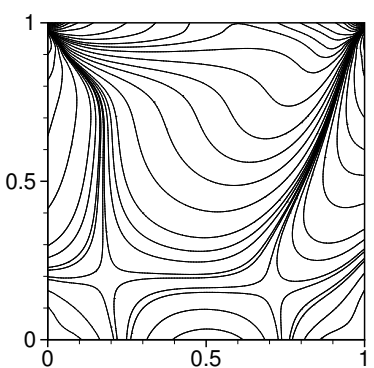

(b) contours of vorticity

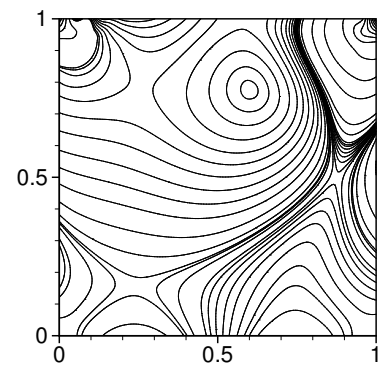

(c) contours of pressure

Figure 3. Navier-Stokes driven cavity flow $(R e=100)$

The third example is the backward facing step flow. In this example, it is known that the recirculation region attached to the step is determined by the Reynolds number and the scale of the step. The Reynolds numbers considered are 50, 100, 150 and 200 for the case of equal step, i.e., the geometric size of inflow and the size of step are same. Here, the interest is the size of the recirculation region and the reattachment point of the flow. The result in Figure 4 shows good agreement with previous results $[2,24]$. In the numerical calculation, uniform node sets with $(7841,2021)$ numbers are used for the velocity and the pressure respectively. The boundary condition is given by $u=4 y(1-y)$ and $v=0$ on the inlet $(x=-1)$, while the outlet boundary condition is given by $\frac{\partial u}{\partial n}=0$ and $\frac{\partial v}{\partial n}=0$ on $x=9$, where $n$ is the outward normal vector on the outlet. On the wall, no-slip condition is assigned, i.e., $u=0$ and $v=0$.

As the last example, the Navier-Stokes flow around a circular cylinder in duct is implemented. The emphasis in this example is on calculating viscous flow in a complicated flow domain with a point collocation scheme. The domain has properties of concavity and convexity. Node distributions for the velocity and the pressure are shown in Figure 5. For efficiency of calculation, it is used that the dilation function which is mentioned in the Remark 2.3. In this example, we assign the no-slip condition for velocity $\mathbf{u}=(u, v)$ to the walls (duct $y= \pm 1$ and cylinder $\left.x^{2}+y^{2}=\left(\frac{1}{2}\right)^{2}\right)$ and assume $u=1-y^{2}$ and $v=0$ on the inlet $(x=-2)$. The boundary condition on the outlet $(x=10)$ is assigned as $\frac{\partial u}{\partial n}=0$ and $\frac{\partial v}{\partial n}=0$, where $n$ is the outward normal vector on the outlet. 


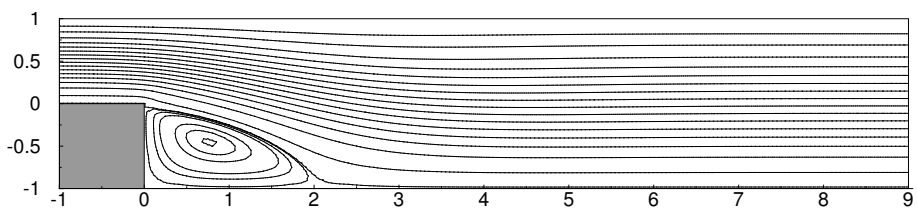

(a) $R e=50$

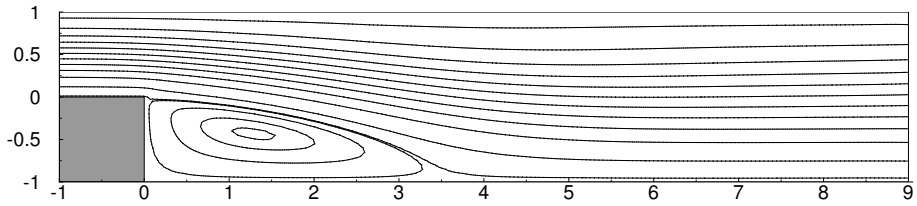

(b) $R e=100$

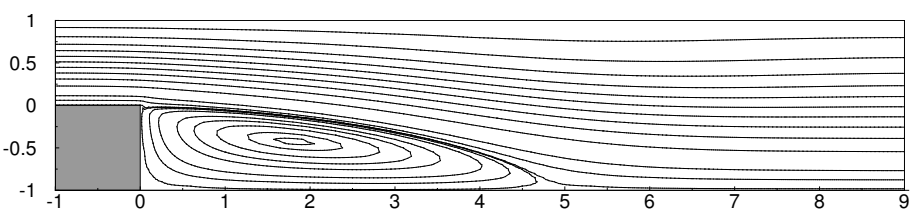

(c) $R e=150$

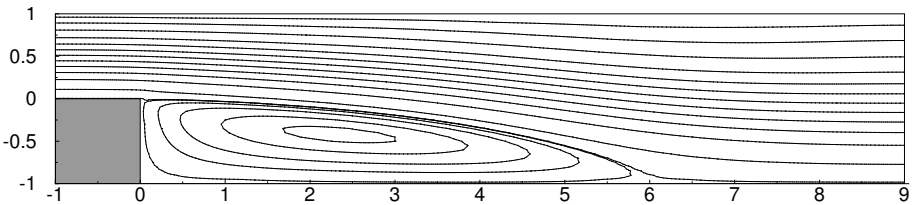

(d) $R e=200$

Figure 4. Stream lines of Navier-Stokes backward-facing step flow

Three plots are in Figure 6. They are the plot of the streamlines, the contour lines of the vorticity and the contour lines of the pressure for the incompressible Navier-Stokes flow at $R e=100$.

\section{Conclusions}

New point collocation scheme for the stationary incompressible Navier-Stokes equations by FMLSRK are proposed. The FMLSRK is a meshfree method which uses approximated derivatives from the local approximation operator. It is highly efficient, since it does not require direct calculations of derivatives for shape functions. The proposed scheme is stable in the sense that the numerical solution of the scheme is the minimizer of the square residual functional. And 


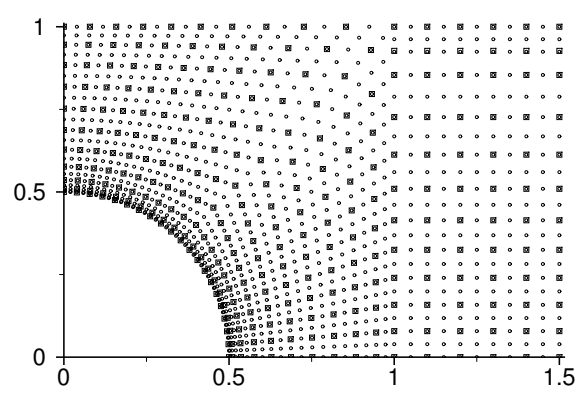

Figure 5. Node distributions for duct flow: velocity node(o) and pressure node $(\square)$

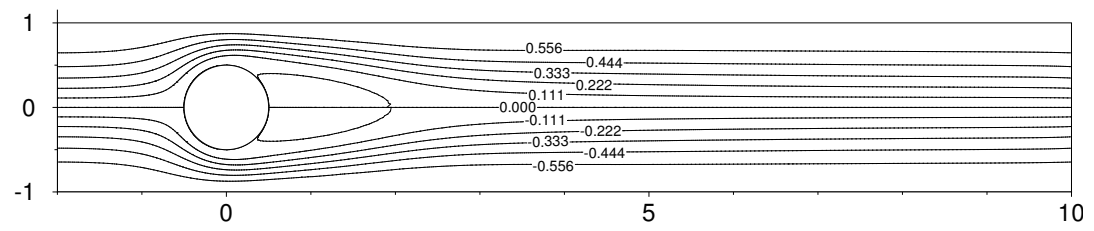

(a) streamlines

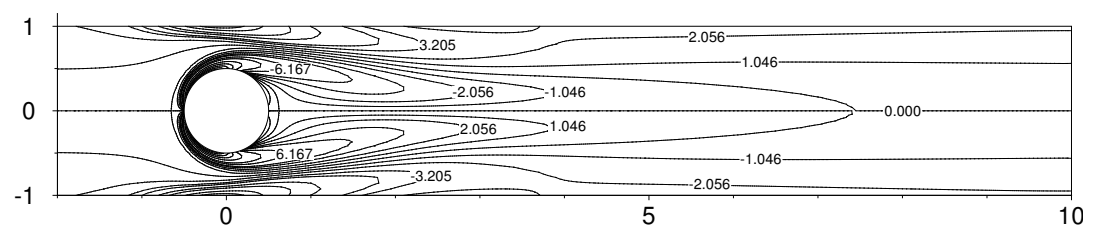

(b) contours of vorticity

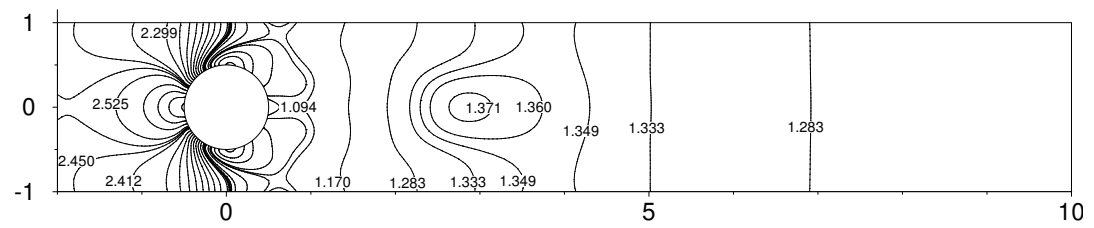

(c) contours of pressure

FiguRE 6. Navier-Stokes flow passing around a circular cylinder in duct $(R e=100)$

resulting stiffness matrix of the scheme is symmetric positive definite even though the given problem does not have symmetric property. 
Through numerical examples, the proposed scheme is validated successfully. The convergence rate of numerical solution to the true solution depends on the consistency order. It is validated numerically that the scheme is applicable even for problems on the complicated geometry.

\section{References}

[1] N. R. Aluru, A point collocation method based on reproducing kernel approximations, Int. J. Numer. Methods Engng. 47 (2000), 1083-1121.

[2] B. F. Armaly, F. Durst, and J. C. Pereira, Experimental and theoritical investigation of backward-facing step flow, J. Fluid Mech. 127 (1983), 473-496.

[3] S. N. Atluri, H. G. Kim, and J. Y. Cho, A critical assessment of the truly Meshless Local Petrov-Galerkin (MLPG) methods, Comput. Mech. 24 (1999), 348-372.

[4] H. J. Choe, D. W. Kim, H. H. Kim, and Y. S. Kim, Meshless method for the stationary incompressible Navier-Stokes equations, Discrete Contin. Dyn. Syst. Ser. B 1 (2001), no. $4,495-526$.

[5] H. J. Choe, D. W. Kim, and Y. S. Kim, Meshfree method for the non-stationary incompressible Navier-Stokes equations, Discrete Contin. Dyn. Syst. Ser. B 6 (2006), no. 1, $17-39$.

[6] C. A. Duarte and J. T. Oden, An h-p adaptive method using clouds, Comput. Methods Appl. Mech. Engrg. 139 (1996), no. 1-4, 237-262.

[7] J. Fürst and T. Sonar, On meshless collocation approximations of conservation laws: Preliminary investigations on positive schemes and dissipation models, Z. Angew. Math. Mech. 81 (2001), no. 6, 403-415.

[8] R. A. Gingold and J. J. Monaghan, Smoothed Particle Hydrodynamics: theory and application to non-spherical stars, Monthly Notices of the Royal Astronomical Society 181 (1977), 275-389.

[9] F. C. Günther and W. K. Liu, Implementation of boundary conditions for meshless methods, Comput. Methods Appl. Mech. Engrg. 163 (1998), no. 1-4, 205-230.

[10] D. W. Kim and H. K. Kim, Point collocation method based on the FMLSRK approximation for electromagnetic field analysis, IEEE Trans. on Magnetics 40 (2004), 1029-1032.

[11] D. W. Kim and Y. S. Kim, Point collocation methods using the fast moving least square reproducing kernel approximation, Internat. J. Numer. Methods Engrg. 56 (2003), no. $10,1445-1464$.

[12] Y. S. Kim, D. W. Kim, S. Jun, and J. H. Lee, Meshfree point collocation method for the stream-vorticity formulation of $2 D$ incompressible Navier-Stokes equations, Comput. Methods Appl. Mech. Engng. 196 (2007), no. 33-34, 3095-3109.

[13] S. Li and W. K. Liu, Synchronized reproducing kernel interpolation via multiple wavelet expansion, Comput. Mech. 21 (1998), 28-47.

[14] _ Reproducing kernel hierarchical partition of unity. I. Formulation and theory, Internat. J. Numer. Methods Engrg. 45 (1999), no. 3, 251-288.

[15] _ Reproducing kernel hierarchical partition of unity. II. Applications, Internat. J. Numer. Methods Engrg. 45 (1999), no. 3, 289-317.

[16] W. K. Liu, S. Jun, S. Li, J. Adee, and T. Belytschko, Reproducing kernel particle methods for structural dynamics, Internat. J. Numer. Methods Engrg. 38 (1995), no. 10, 1655-1679.

[17] W. K. Liu, S. Jun, and Y. F. Zhang, Reproducing kernel particle methods, Internat. J. Numer. Methods Fluids 20 (1995), no. 8-9, 1081-1106.

[18] W. K. Liu, S. Li, and T. Belytschko, Moving least-square reproducing kernel methods. I. Methodology and convergence, Comput. Methods Appl. Mech. Engrg. 143 (1997), no. $1-2,113-154$. 
A POINT COLLOCATION SCHEME FOR THE NAVIER-STOKES EQUATIONS 1751

[19] Y. Y. Lu, T. Belytschko, and L. Gu, A new implementation of the element free Galerkin method, Comput. Methods Appl. Mech. Engrg. 113 (1994), no. 3-4, 397-414.

[20] Y. Luo and U. Häussler-Combe, A generalized finite-difference method based on minimizing global residual, Comput. Methods Appl. Mech. Engrg. 191 (2002), no. 13-14, 1421-1438.

[21] J. M. Melenk and I. Babuška, The partition of unity finite element method: basic theory and applications, Comput. Methods Appl. Mech. Engrg. 139 (1996), no. 1-4, 289-314.

[22] B. Nayroles, G. Touzot, and P. Villon, Generalizing the finite element method: diffuse approximation and diffuse elements, Comput. Mech. 10 (1992), 307-318.

[23] X. Zhang, X. Liu, K. Song, and M. W. Lu, Least-square collocation meshless method, Internat. J. Numer. Methods Engrg. 51 (2001), no. 9, 1089-1100.

[24] Y. Zhao and B. Zhang, A high-order characteristics upwind FV method for incompressible flow and heat transfer simulation on unstructured grids, Comput. Methods Appl. Mech. Engrg. 190 (2000), no. 5-7, 733-756.

[25] T. Zhu, J. Zhang, and S. N. Atluri, A meshless local boundary integral equation (LBIE) method for solving nonlinear problems, Comput. Mech. 22 (1998), no. 2, 174-186.

Department of Financial EngineERing

AJou UnIVERsity

Suwon 443-749, Korea

E-mail address: yskim67@ajou.ac.kr 